\title{
Investigation and Improvement of Stall Characteristic of High-Lift Configuration without Slats
}

\author{
Zhao Yang $\mathbb{D}^{1}{ }^{1}$ Jie Li $\mathbb{D}^{1},{ }^{1}$ Jing Jin, ${ }^{2}$ Heng Zhang, ${ }^{1}$ and Youxu Jiang ${ }^{1}$ \\ ${ }^{1}$ School of Aeronautics, Northwestern Polytechnical University, 710072 Xi'an, China \\ ${ }^{2}$ Shanghai Aircraft Design and Research Institute, Commercial Aircraft Corporation of China Ltd., 200126 Shanghai, China \\ Correspondence should be addressed to Jie Li; lijieruihao@nwpu.edu.cn
}

Received 11 September 2019; Accepted 31 October 2019; Published 26 November 2019

Academic Editor: Hao J. Xia

Copyright (c) 2019 Zhao Yang et al. This is an open access article distributed under the Creative Commons Attribution License, which permits unrestricted use, distribution, and reproduction in any medium, provided the original work is properly cited.

\begin{abstract}
In order to simplify the manufacturing process or because of the limitation of the propulsion system, business jet, small civil airplane, and turboprop aircraft are always designed without leading-edge slats, which poses a great challenge to the flight safety during takeoff and landing. Focusing on the low-speed stall and poststall conditions, we investigated the aerodynamic characteristics and flow mechanism of high-lift configuration without slats using an improved delayed detached eddy simulation (IDDES) model which is validated by numerical simulations of the Common Research Model (CRM). Based on the analysis of the calculated results, conclusion can be made that the stall behavior of the configurations is directly related to the onset and evaluation of flow separation on the suction side. And through further research, an efficient evaluation method that is capable of qualitatively predicting the stall performance of two-element high-lift configuration by stall angle distribution of wing sections is proposed. By using the evaluation method, together with design rules summarized from the present study, high-lift configuration with mild-stall characteristic can be obtained in the preliminary stage of design.
\end{abstract}

\section{Introduction}

Stall behavior of aircraft is one of the most important aspects that should be emphasized in the preliminary design stage as it directly affects the safety and maneuverability of aircraft during takeoff and landing. One commonly used approach of delaying wing stall is by installing a well-designed leading-edge slat to effectively lower adverse pressure gradients and suppress separation on the upper side as the angle of attack increases [1-3]. In addition to the installation of a slat, aerodynamic accessories such as stall strips, wing fences, and vortex generators can be utilized for improving the local stall behavior without major configuration modifications in the final stages of design [4]. However, their effects are highly dependent on aircraft configuration and must be further validated by a flight test program [5]. Therefore, for business jet, turboprop aircraft, and small civil airplane which are unsuitable for the installation of leading-edge slats, it is particularly important to equip the high-lift configuration with mild-stall characteristics to enhance the flight safety of aircraft.
In a stall situation, the aerodynamic forces of aircraft show obvious nonlinear characteristics due to the appearance of massive flow separation on the suction side and the flow physics at poststall conditions are not well understood neither by experimental nor computational research until now. A large number of theoretical and experimental investigations of stall and aerodynamic characteristics have been carried out at the end of the 20th century and are very helpful to our research in this paper. Wind tunnel and full-scale flight tests were performed on a general aviation configuration by Johnson et al. [6] and Meyer et al. [7], respectively, to analyze the influence of leading-edge modification on the flow separation state and stall pattern of the wing. Winkelmann et al. [8] investigated the poststall characteristics of two rectangular planform wings with leading-edge modifications through a series of low-speed wing tunnel tests. Anderson [9] reviewed the primary factors which had affected stall/spin behavior of general aviation aircraft over the history of flight and proposed several prospects for aerodynamic improvements in stall/spin. 
TABLE 1: Application of the RANS/LES method in complex configurations.

\begin{tabular}{lcccccc}
\hline \multirow{2}{*}{ Configuration } & \multicolumn{3}{c}{ Calculation parameters } & \multirow{2}{*}{ Calculation method } & Type of grid & Grid \\
& $\operatorname{Re}\left(\times 10^{6}\right)$ & $\mathrm{Ma}$ & $\alpha$ & DES6er of grids $\left(\times 10^{6}\right)$ \\
\hline F-15E [10] & 13.6 & 0.3 & $65^{\circ}$ & Unstructured & $5.9 / 10$ \\
F-18C [11] & 13.9 & 0.275 & $30^{\circ}$ & DES97 & Unstructured & 6.2 \\
F/A-18E [12] & 3.98 & 0.9 & $9^{\circ}$ & DES97 & Unstructured & 7.3 \\
F-16XL [13] & 32.2 & 0.24 & $19.8^{\circ}$ & RANS-LES & Unstructured & 4.5 \\
NASA TN D-712 [14, 15] & 7.5 & 0.9 & $12.5^{\circ} / 26.2^{\circ}$ & SST-DDES & Structured & 2.2 \\
NASA CRM [16-18] & 11.6 & 0.25 & $16^{\circ} / 18^{\circ}$ & SA-DDES & Unstructured & 51 \\
DLR-F11 [19] & - & 0.175 & $21^{\circ} / 22.4^{\circ}$ & DES97 & Structured & $11 / 16$ \\
\hline
\end{tabular}

Since the 21st century, with the development of the hybrid RANS/LES method, CFD has gradually become a common research tool for stall problems as the hybrid method can greatly improve the simulation accuracy of turbulent flow structures in separation regions without a significant increase in computational resources. The investigation and improvement of the RANS/LES hybrid method have always been the focus of research. Over the last two decades, many new branches of hybrid methods with better computational performance have been developed and applied for numerical prediction of separated flow of complex aircraft configurations by researchers as listed in Table 1. Forsythe et al. [10] used the DES method for the first time to analyze the aerodynamic characteristics of F-15E in deep stall at a high angle of attack and compared the results with those obtained from the RANS method. The results showed that DES can capture the unsteady three-dimensional flow structures in the separation wake, and the predicted aerodynamic coefficients agreed better with the experimental results than that of the RANS method. Morton et al. [11] first combined an adaptive mesh refinement technique with the DES method to calculate the separated flow of F-18C at a 30 degree angle of attack. The calculated results indicated that the lift coefficient and the location of vortex breakdown obtained by DES are qualitatively consistent with the experimental data. Then, in 2005, Forsythe and Woodson [12] analyzed the abrupt stall problem of F/A-18E configuration by the same way and the results further showed that the adaptive mesh refinement technique improved the prediction accuracy of DES. Tomac et al. [13] used the RANS/LES method which combines the B-L turbulence model with the Smagorinsky model to simulate the F-16XL configuration in lowspeed at a high angle of attack. Because of the interference effect of vortex shedding, the result obtained through the hybrid method was obviously better than that of the RANS method on the outboard region of the wing. Fu et al. and Xiao et al. $[14,15]$ used RANS, DES, and DDES methods based on a two-equation $k$ - $\omega$ SST turbulence model to calculate the NASA TN D-712 wing-body configuration. After comparing the calculated and experimental results, the authors concluded that the DDES calculation results were more reliable and could reveal more detailed flow information and vortex structures than those of RANS. What is more, Waldmann et al. [16], Lutz et al. [17], and Zimmermann et al. [18] numerically investigated the influence of unsteady wake on the tail of NASA CRM wing-body-tail configuration under poststall condition by the DDES method based on a TAU solver. Escobar et al. [19] made use of the DES97 method in ANSYS Fluent to make a detailed analysis of the flow around the DLR-F11 high-lift configuration.

This paper starts with the introduction of stall characteristic investigations and several applications of the hybrid RANS/LES method in aeronautical problems. Section 2.1 provides a description of the newly developed IDDES model, and Section 2.2 proposes a validation of the numerical approach through the simulation of the Common Research Model. Numerical simulations of two high-lift configurations without slats under pre- and poststall conditions are performed using the IDDES method in Section 3. Sections 3.1 and 3.2 give the geometry and grids used in the computation, respectively. The physics of the onset and evolution of flow separations as well as their effects on stall characteristics are discussed in Section 3.3. Then, an efficient method that is capable of predicting and improving the stall performance of wings in the preliminary stage of design is proposed in Section 4.1, and several guidelines for the design of mild-stall high-lift configuration without slats are summarized in Section 4.2 .

\section{Numerical Methodology}

2.1. IDDES Method. In order to deal with the log-layer mismatch (LLM) phenomenon which usually occurs in a DES model, Shur et al. [20] developed the IDDES method through further improvement of DDES. Compared to the DES/DDES model, IDDES provides shielding against Grid Induced Separation (GIS) and allows the model to run in the wallmodeled LES (WMLES) mode, providing a possibility to simulate an unsteady mode of near-wall boundary layers.

The IDDES method has two branches: DDES and WMLES. The choice between RANS and LES regions in IDDES is dictated in Equation (1).

$$
\begin{aligned}
l_{\mathrm{hyb}} & =l_{\mathrm{IDDES}}=\tilde{f}_{d} \cdot\left(1+f_{e}\right) \cdot l_{\mathrm{RANS}}+\left(1-\tilde{f}_{d}\right) \cdot l_{\mathrm{LES}}, \\
l_{\mathrm{LES}} & =C_{\mathrm{DES}} \cdot \Delta_{\mathrm{IDDES}}, \\
\Delta_{\mathrm{IDDES}} & =\min \left[\max \left(C_{\mathrm{w}} d_{\mathrm{w}}, C_{\mathrm{w}} h_{\mathrm{max}}, h_{\mathrm{wn}}\right), h_{\mathrm{max}}\right], \\
h_{\max } & =\max (\Delta x, \Delta y, \Delta z),
\end{aligned}
$$

where $\Delta x, \Delta y$, and $\Delta z$ are the grid spacing in the $x, y$, and $z$ directions, $C_{\mathrm{w}}=0.15$ is an empirical constant, $d_{\mathrm{w}}$ is the 
wall distance, and $h_{\mathrm{wn}}$ is the grid step in the wall-normal direction.

When $f_{e}=0$, the hybrid length scale turns out to be the DDES mode:

$$
l_{\mathrm{IDDES}}=l_{\mathrm{DDES}}=\tilde{f}_{d} \cdot l_{\mathrm{RANS}}+\left(1-\tilde{f}_{d}\right) \cdot l_{\mathrm{LES}} .
$$

When $f_{e}>0$ and $\tilde{f}_{d}=f_{B}$, the hybrid length scale turns out to be the WMLES mode:

$$
l_{\mathrm{IDDES}}=l_{\mathrm{WMLES}}=f_{B} \cdot\left(1+f_{e}\right) \cdot l_{\mathrm{RANS}}+\left(1-f_{B}\right) \cdot l_{\mathrm{LES}} \cdot
$$

The $\tilde{f}_{d}$ function contains two additional blending functions that represent a DDES type of shielding $f_{d t}$ and one suitable for wall-modelled large eddy simulation (WMLES) $f_{B}$ :

$$
\begin{aligned}
\tilde{f}_{d} & =\max \left\{\left(1-f_{d t}\right), f_{B}\right\}, \\
f_{d t} & =1-\tanh \left[\left(C_{d t 1} \cdot r_{d t}\right)_{d t 2}\right], \\
r_{d t} & =\frac{v_{t}}{\kappa^{2} d_{\mathrm{w}}^{2} \sqrt{0.5\left(S^{2}+\Omega^{2}\right)}},
\end{aligned}
$$

where $C_{d t 1}=8, C_{d t 2}=3, S$ is the strain rate tensor, and $\Omega$ is the curl tensor.

The empirical blending function $f_{B}$ is in the form of Equation (5). It varies from 0 to 1 and provides rapid switching of the model from the RANS mode $\left(f_{B}=1.0\right)$ to the LES mode $\left(f_{B}=0\right)$ within the range of wall distance $0.5 h_{\max }<$ $d_{\mathrm{w}}<h_{\max }$.

$$
f_{B}=\min \left\{2 \exp \left(-9 \alpha^{2}\right), 1.0\right\}, \quad \alpha=0.25-\left(\frac{d_{\mathrm{w}}}{h_{\max }}\right) .
$$

The second empirical function $f_{e}$ is aimed at preventing the excessive reduction of the RANS Reynolds stresses observed in the interaction of the RANS and LES regions in the vicinity of their interface. It is intended to eliminate the LLM problem.

$$
\begin{aligned}
f_{e} & =f_{e 2} \cdot \max \left(\left(f_{e 1}-1.0\right), 0.0\right), \\
f_{e 1} & = \begin{cases}2 \exp \left(-9 \alpha^{2}\right), & \text { if } \alpha<0, \\
2 \exp \left(-11.09 \alpha^{2}\right), & \text { if } \alpha \geq 0,\end{cases} \\
f_{e 2} & =1.0-\max \left(f_{t}, f_{l}\right), \\
f_{t} & =\tanh \left(\left(C_{t}^{2} \cdot r_{d t}\right)^{3}\right), \\
f_{l} & =\tanh \left(\left(C_{l}^{2} \cdot r_{d l}\right)^{10}\right), \\
r_{d l} & =\frac{v}{\kappa^{2} d_{\mathrm{w}}^{2} \sqrt{0.5\left(S^{2}+\Omega^{2}\right)}} .
\end{aligned}
$$

The values of constants $C_{t}$ and $C_{l}$ are selected according to the turbulence model used in the IDDES calculation. And for the SST model, $C_{l}=5.0$ and $C_{t}=1.87$.

\subsection{Validation of the Numerical Approach}

2.2.1. Geometric Configuration. The test case is conducted on the conceptual wing/body/tail (WBT) configuration of the Common Research Model (CRM) for the study of flow phenomena occurring at stall conditions. The CRM is a typical wide-body commercial transport aircraft configuration with a contemporary supercritical transonic wing. It is designed for a cruise Mach number of $\mathrm{Ma}=0.85$ and a corresponding design lift coefficient of $\mathrm{CL}=0.5$ [21]. The CRM configuration has been used as the research model for the fourth and fifth AIAA drag prediction workshop (DPW) and has been tested in different wind tunnels around the world for various purposes. In this paper, the model and experimental data from the European Transonic Wind Tunnel (ETW) are selected for computational fluid dynamics (CFD) computation and result verification [22]. In the ETW experiment, a $2.7 \%$ scaled model was manufactured in a $1 \mathrm{~g}$ design shape, which represents the deformation in level flight [23]. The geometric model with aeroelastic deformation is shown in Figure 1, and the main parameters are listed as follows:

(i) Incidence of the horizontal tail is $0^{\circ}$

(ii) Length of the fuselage is $l=1.695 \mathrm{~m}$

(iii) Half span of the wing is $b=0.793 \mathrm{~m}$

(iv) Length of the mean aerodynamic chord of the clean wing is $c=0.189 \mathrm{~m}$

(v) Reference area is $s=0.28 \mathrm{~m}^{2}$

2.2.2. Computational Grid. The structured multiblock grid of the WBT configuration is generated by ICEM CFD in the present study. In the near-wall region, the O-type topology is used to densify the boundary layer while the flow field is filled with an H-type grid. The far-field boundary is at a distance of 150 times the length of the mean aerodynamic chord from the aircraft in three directions. The resolution of the grid in the region above the wing and extending a mean aerodynamic chord to the wake are on the order of $3.5 \%$ of the reference chord length in all dimensions. The first cell height for the normal wall grids is set to $1 \times 10^{-6}$ to ensure $y^{+} \approx 1$, and the total mesh size of the half model is about $65.0 \times 10^{6}$. The details of the blocking structure and surface grid are shown in Figure 2.

2.2.3. Computation Setup. The CFD computations were performed using an in-house structured code [24] with a cell-central finite volume formulation in this paper. Spatial discretization of the viscous terms is done using a secondorder central differencing scheme, and the convective and pressure terms are discretized with a third-order flux difference splitting method of Roe's scheme. The solution is advanced in time by the implicit lower-upper symmetric Gauss-Seidel (LU-SGS) scheme with subiteration in pseudo 


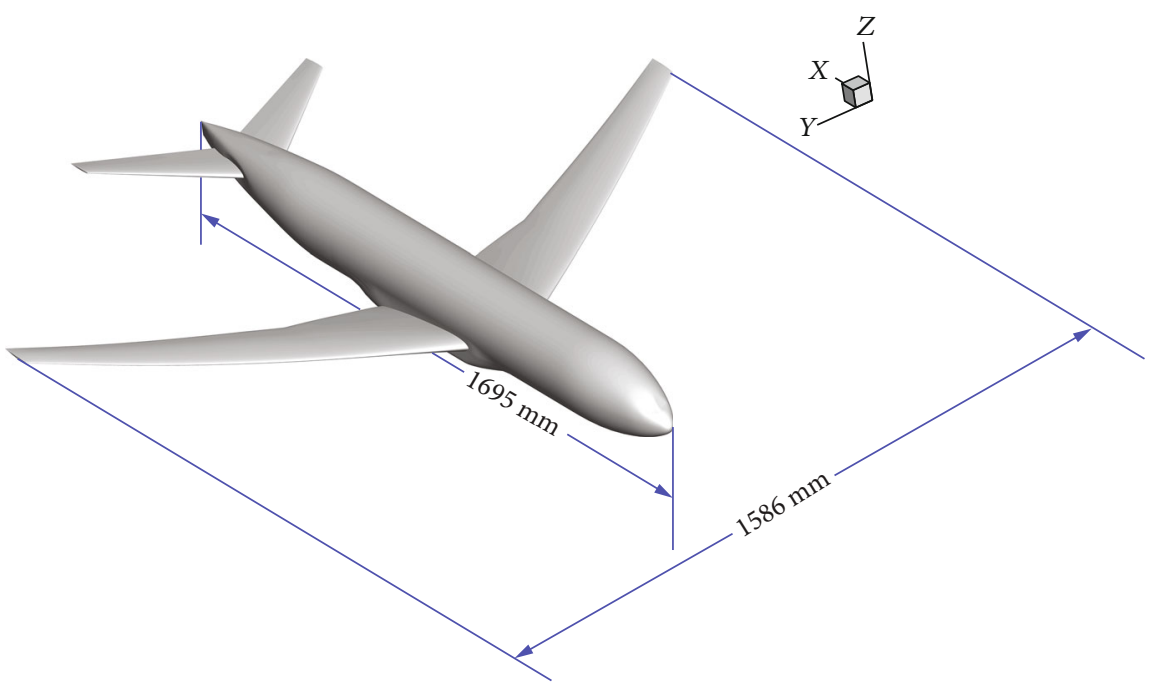

Figure 1: Geometry of the CRM in the ETW experiment.
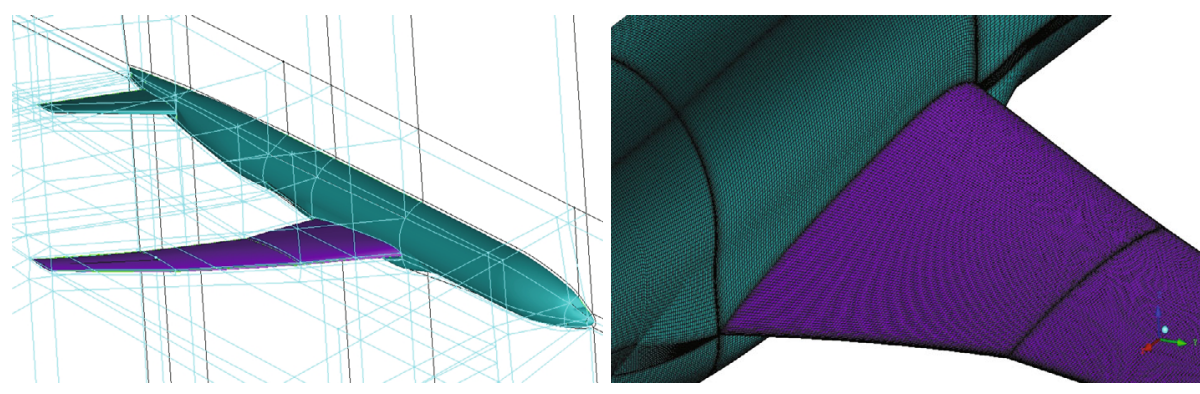

FIGURE 2: Blocking structure and surface grid of the CRM.

time. The URANS and IDDES methods based on the $k-\omega$ SST turbulence model were introduced to this simulation. The time step of unsteady calculation is set at $\Delta t *=0.01$, and the corresponding physical time step is about $\Delta t=8.8 \times$ $10^{-6} \mathrm{~s}$. For the investigations presented in this paper, 40000 time steps were recorded and at least 25 subiterations are carried out in each time step to guarantee a sufficient convergence behavior. The computations were performed using 4 nodes with 24 cores per node by utilizing the computational resources from the high-performance cluster "TIANHE," and the approximate total runtime for each operating point was 280 hours.

2.2.4. Results and Discussion. The total force coefficients calculated by RANS/URANS/IDDES, respectively, are compared to the measured data of ETW in Figure 3. Calculation and test conditions are given in Table 2 [18]. RANS is used over the entire angle of attack ranging up to $18^{\circ}$ while URANS and IDDES are only applied for the pre- and poststall conditions including $10^{\circ}, 12^{\circ}, 14^{\circ}, 16^{\circ}$, and $18^{\circ}$. The steady results of RANS only show good agreement with the experimental data in a linear region before $12^{\circ}$. The timeaveraged lift coefficients predicted by URANS are obviously lower than the measurement results at $12^{\circ}$ and $14^{\circ}$, and the maximum deviation occurs at $14^{\circ}$ with a relative error of $18 \%$. On the contrary, the results of drag coefficients at the two conditions are much higher than the experimental value.
The time-averaged forces from IDDES match the measurement results better than those from URANS at all the abovementioned operating points, especially at $16^{\circ}$ and $18^{\circ}$. Lutz et al. [17] and Zimmermann et al. [18] have performed URANS/DDES simulations of CRM at $16^{\circ}$ and $18^{\circ}$, and their results and conclusions are basically consistent with those in this paper.

The shape of the polar in Figure 3 indicates a large drop of lift accompanied by an equally significant increase in drag in the vicinity of $16^{\circ}$, revealing a major change of flow topology and stall mechanism at this point. Figure 4 shows the time-averaged surface pressure contour and streamlines (averaged over 2000 steps) calculated by the URANS and IDDES methods. It can be seen from the comparisons that obvious differences exist between the two methods in predicting the surface streamline patterns and low-pressure region of the wing at $16^{\circ}$ and $18^{\circ}$. The IDDES method is able to capture the crossflow on the wing. The crossflow from the leading edge meets the separation flow rolled up from the trailing edge, forming a dividing line as indicated by the pink arrow in Figure 4. In comparison, URANS predicts complete separation without reattachment on the upper surface. Hence, separation on the suction side calculated by IDDES is weaker than that calculated by URANS. The time-averaged pressure distributions in Figures 5(a) and 5(b) also visualize the differences between the URANS and IDDES results. It becomes obvious that the discrepancies 


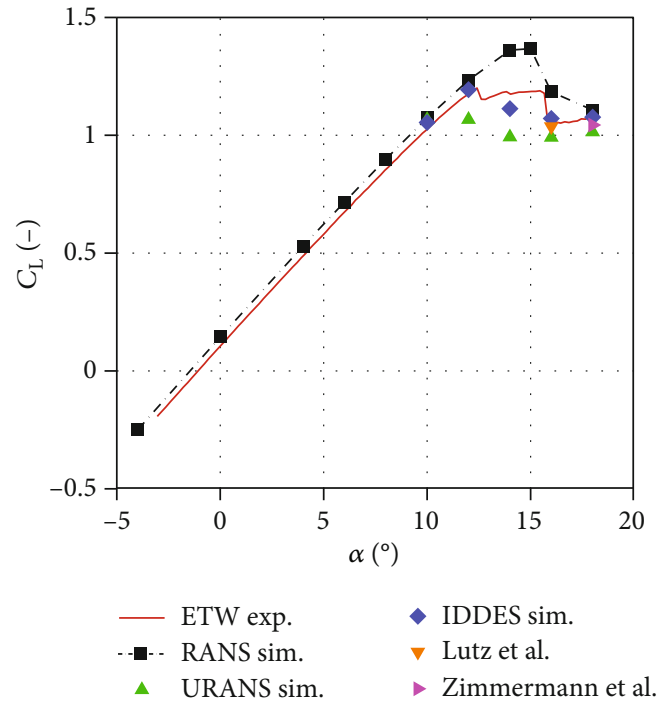

(a) Lift coefficient

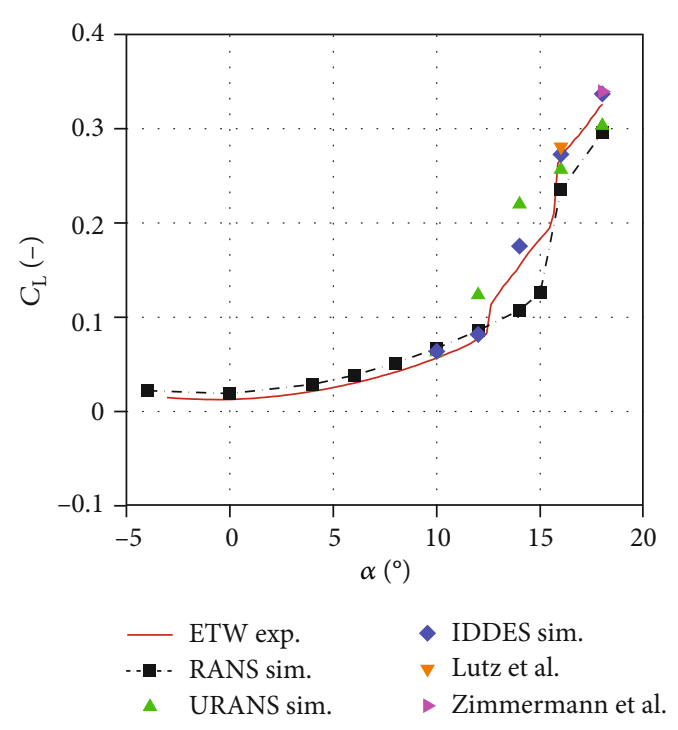

(b) Drag coefficient

FIGURE 3: RANS/URANS/IDDES simulation results in comparison to experimental data of ETW measurements.

TABlE 2: Test conditions in the ETW for low-speed stall measurements [18].

\begin{tabular}{lcccc}
\hline$\alpha\left(^{\circ}\right)$ & $\mathrm{Ma}$ & $T_{\text {tot }}(\mathrm{K})$ & $p(\mathrm{kPa})$ & $\operatorname{Re}\left(10^{6}\right)$ \\
\hline$-3^{\circ} \sim 18^{\circ}$ & 0.25 & 115 & 303 & 11.6 \\
\hline
\end{tabular}

are located in the inboard region of the wing, where a complex flow situation and an influence of the fuselage are expected. The IDDES model tends to predict the separation at the leading edge and the pressure distribution better, especially at the location $\eta=20.1 \%$.

To sum up, for large-scale separation problems of aircraft, great differences exist between IDDES and URANS results in the flow topology and mechanism which may directly lead to the discrepancy of predicted aerodynamic characteristics. As a high-resolution model, the results of IDDES are always more reliable and convincing. Hence, IDDES can be used as a state-of-the-art CFD tool for the investigation on stall characteristics of complex aircraft configuration to better understand the physics of onset, evolution, and effect of flow separation under poststall conditions.

\section{Calculation and Flow Field Analysis}

Numerical simulations of two two-element high-lift configurations with different geometric characteristics under poststall conditions are performed using the IDDES method. The characterizations of the turbulent flow above the surface and in the wake of the stalled wings are of particular interest. The physics of the onset and evolution of flow separations as well as their effects on aerodynamics characteristics are further studied by using the obtained flow field data.

3.1. Geometric Configuration. Wing-A and Wing-B are both two-element high-lift configurations which are typically used for turboprop aircraft. The two wings have the same layout and are both equipped with a simple Fowler flap which is extended along the whole span and fits well with the trailing edge of each wing. Figure 6(a) shows a sketch of the geometry of the high-lift configuration, and Figure 6(b) depicts the dimensionless $x$ and $y$ coordinates of the front and rear edges of a clean wing in the top view as well as the relative thickness and twist at four spanwise positions $(\eta=0 \%, 32 \%, 56 \%$, and $100 \%$ from the wing root to the tip). Comparisons of airfoil profiles of Wing-A and Wing-B at four different sections are demonstrated in Figure 6(c). The geometric characteristics of Wing-A's airfoil profiles are similar to those of highlift airfoils with a large leading-edge radius and camber while the profiles of Wing-B are closer to laminar airfoils whose leading-edge radii are smaller and the maximum thickness of the airfoil typically locate at the rearward position.

The main geometric features of Wing- $\mathrm{A}$ and Wing- $\mathrm{B}$ are listed as follows:

(1) Half span of the wing is $b=13.5 \mathrm{~m}$

(2) Length of the clean wing's root is $c_{\mathrm{r}}=3.375 \mathrm{~m}$, and length of the clean wing's tip is $c_{\mathrm{t}}=1.35 \mathrm{~m}$

(3) Length of the mean aerodynamic chord of the clean wing is $c=2.45 \mathrm{~m}$

(4) Aspect ratio of the clean wing is $\lambda=5.5$

(5) Reference area is $s=33.075 \mathrm{~m}^{2}$

(6) Coordinates of the moment center are $x_{\mathrm{mc}}=0$, $y_{\mathrm{mc}}=0$, and $z_{\mathrm{mc}}=0$

(7) Sweep angle of the leading edge is $\chi_{0}=5^{\circ}$

The position of the Fowler flap related to the wing geometry is defined by the following parameters:

(8) Fowler flap: $30^{\circ}$ deflection, $2.0 \%$ overlap, and $1.5 \%$ gap 


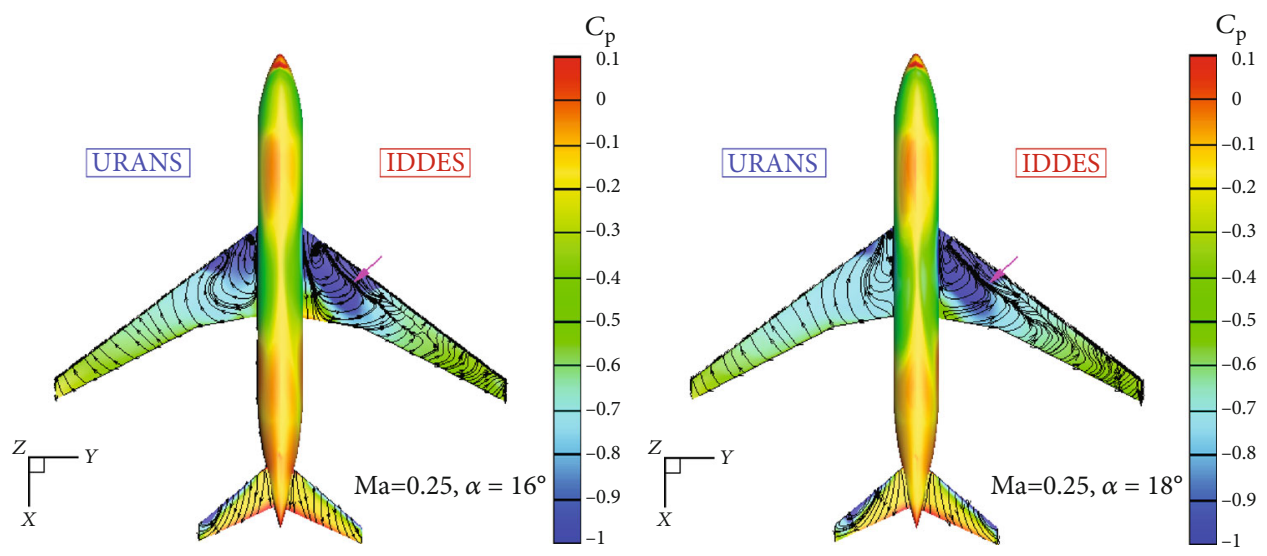

FIGURE 4: Time-averaged pressure contour and streamlines on the wing surface.

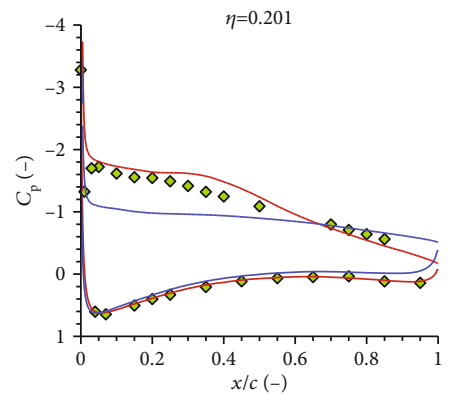
$\diamond \quad$ ETW exp.
IDDES sim.

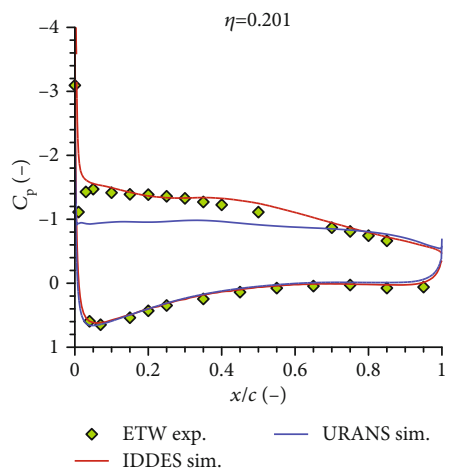

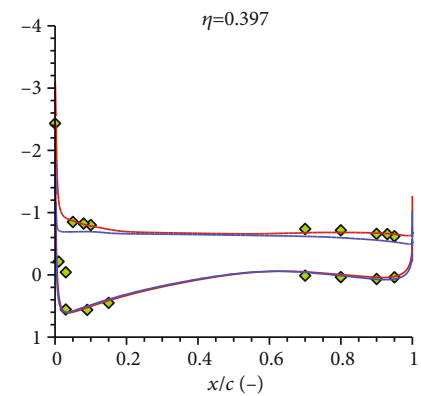

$\diamond \quad$ ETW exp.

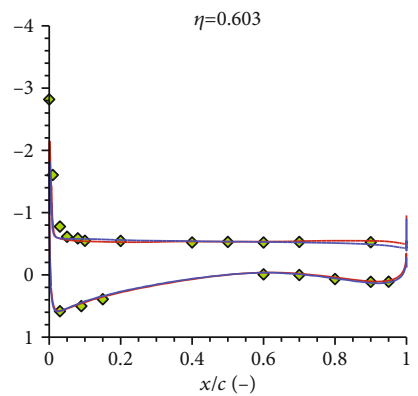

— URANS sim. $\diamond \quad$ ETW exp.
- IDDES sim.

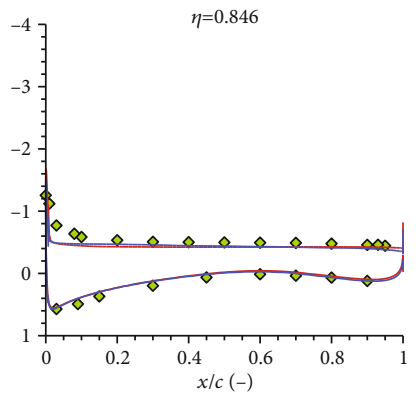

$\diamond \quad$ ETW exp.

(a) $\alpha=16^{\circ}$
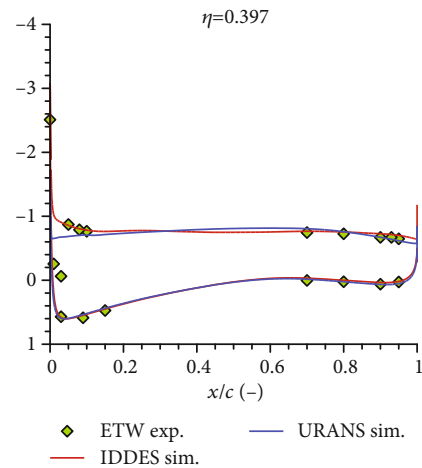

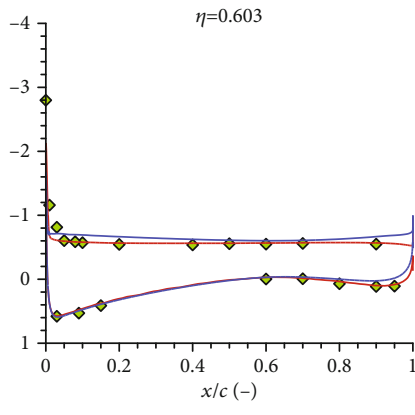

$\diamond \quad$ ETW exp.
$\quad$ IDDES sim.

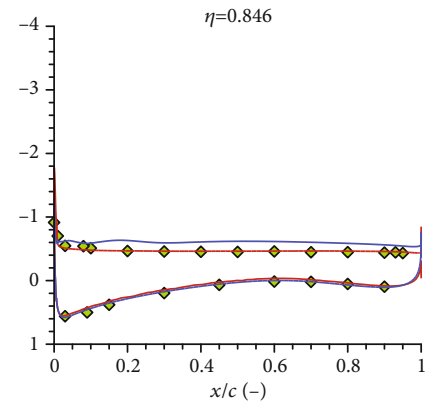

$\diamond \quad$ ETW exp. — URANS sim.

(b) $\alpha=18^{\circ}$

3.2. Computational Grid. The grids used in the present work are fully structured hexahedral meshes generated with ICEM CFD, and the total mesh size is about $47.0 \times$ $10^{6}$. The volume consists of 25 structured layers to ensure a good resolution of the boundary layers. The initial wall spacing is $\Delta h=0.01 \mathrm{~mm}$, such that the height of the first layer meets the criterion of $y^{+} \approx 1$ over the whole surface of the wing and the expansion rate in the wall-normal direction within the structured layers is 1.2 globally. The overall computational domain has an approximate size of $50 c \times 35 c \times 50 c$ in streamwise, spanwise, and vertical directions, respectively. Furthermore, the grids with local refinement in a cuboid region with a size of $2.0 c \times 1.5 b \times 1.0 c$ in each direction $(x, y$, and $z)$ just above the wing and further extending to the wake region behind the flap are created to better resolve vortex structures and flow separation at a high angle of attack. Based on an estimation of the turbulent length-scale results of a SST-RANS simulation, the resolution of the grids in the cuboid region $\Delta x, \Delta y$, and $\Delta z$ is determined. Table 3 shows the parameters for the generated meshes and the details of the surface grid are illustrated in Figure 7.

3.3. Results and Discussion. For this study, simulations have been performed at different angles of attack with the following freestream conditions: 


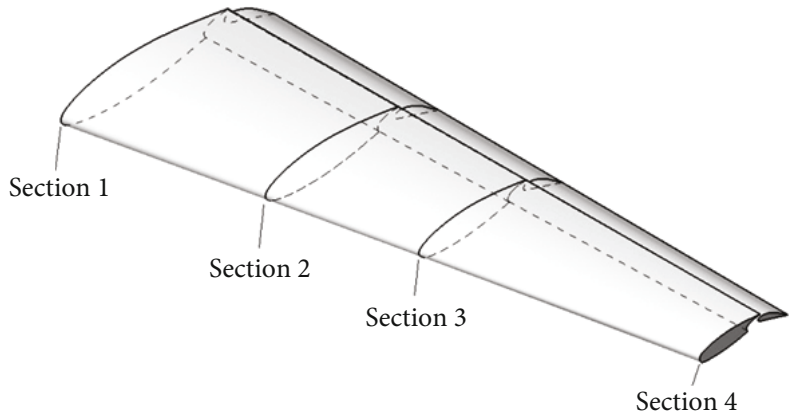

(a)

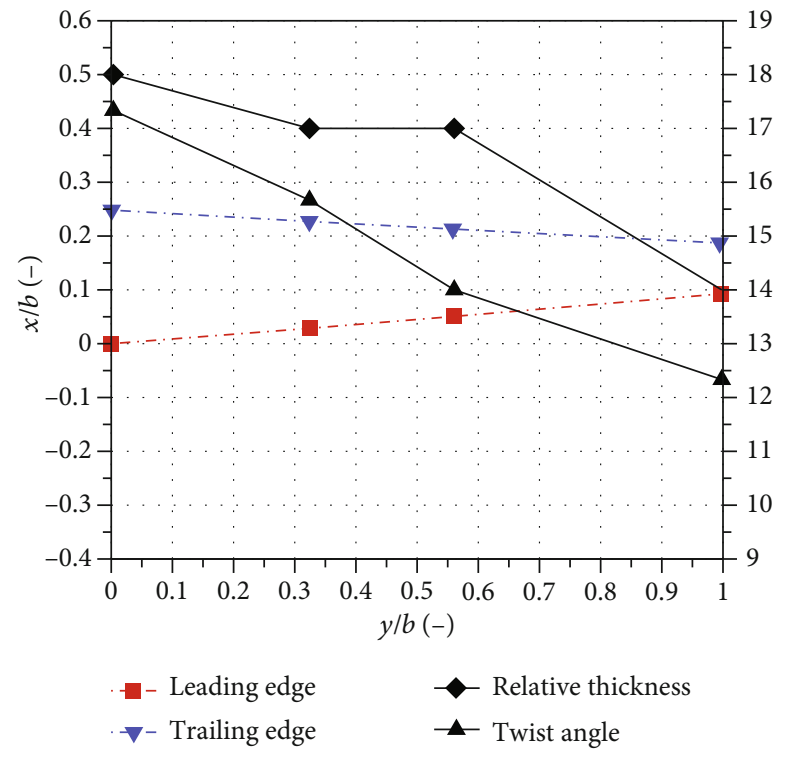

(b)

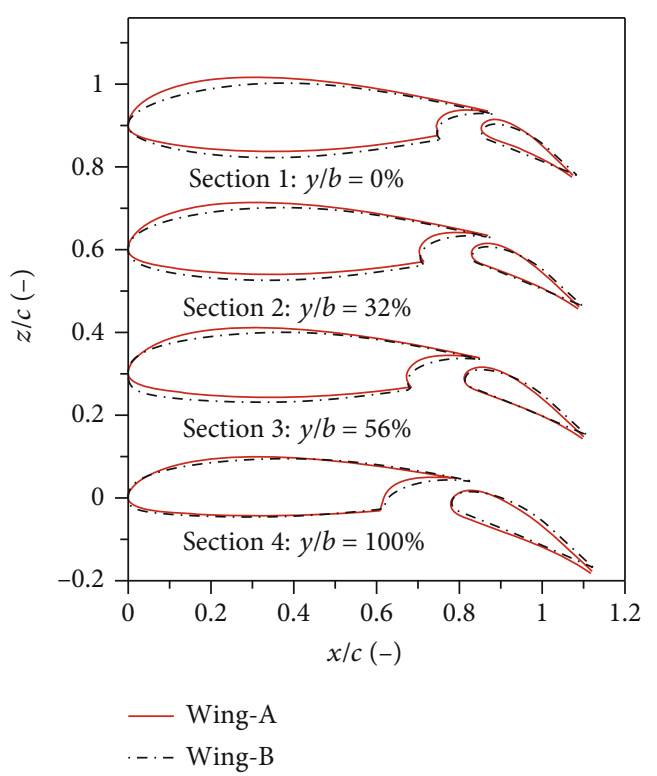

(c)

Figure 6: Geometric features of Wing-A and Wing-B: (a) the geometry; (b) the relative thickness and twist angle of different sections of the clean wing; (c) the profile of each wing section.

TABle 3: Parameter overview of the used meshes.

\begin{tabular}{lccccc}
\hline Parameters & Number of points & $\Delta h$ & $\Delta x / c$ & $\Delta y / c$ & $\Delta z / c$ \\
\hline Value & 47 million & $0.01 \mathrm{~mm}$ & 0.0025 & 0.005 & 0.005 \\
\hline
\end{tabular}

(i) Reynolds number $\mathrm{Re}=1.0 \times 10^{7}$ (based on $c=$ $2.45 \mathrm{~m})$

(ii) Mach number $\mathrm{Ma}=0.2$

(iii) Angle of attack $\alpha=0 / 4 / 8 / 12 / 14 / 16 / 17 / 18 / 19 / 20 /$ $21 / 22$

Two computational approaches were used here: URANS and an IDDES model. In the first step, the preliminary results for all the angles of attack were obtained using the URANS method and it can be seen from the results that the nonlinear phenomena caused by flow separation mainly occur after a 14-degree angle of attack. Hence, the IDDES simulations were restarted from the URANS results for incidences in the range of 16 to $22^{\circ}$. The calculations were obtained using the dimensionless time step of $\Delta t a_{\infty} / c=$ 0.001 , where $a_{\infty}$ is the freestream sound speed. This time step was chosen to attain a CFL number less than one in the domain of interest where the strong separation takes place and 15000 time steps with at least 25 subiterations per step were recorded in all. The computations were performed using 3 nodes with 24 cores per node by utilizing the computational resources from the high-performance cluster "TIANHE," and the approximate total runtime for each operating point was 135 hours.

Figure 8 shows the comparison of the lift and moment characteristics of the two wings. As visible in Figure 8(a), the lift coefficient of Wing-A is always bigger than that of Wing-B until the angle of attack reaches 19 degrees and the difference between them continuously decreases from about 0.3 at $0^{\circ}$ to less than 0.1 at $18^{\circ}$ as the incidence increases. Wing-A and Wing-B achieve maximum lift coefficients of 2.75 and 2.68, respectively, at 18 degrees which is the critical angle of attack for both of them. Then, the lift coefficient of Wing-A encounters a sharp decline after 18 degrees while the change of the lift coefficient of Wing-B turns out to be a relatively gentle process. Wing-A's lift coefficient drops dramatically to 2.57 at 19 degrees and continues to reduce almost linearly to 2.28 at 22 degrees. By contrast, Wing-B's lift coefficient at 19 degrees has little change compared with 


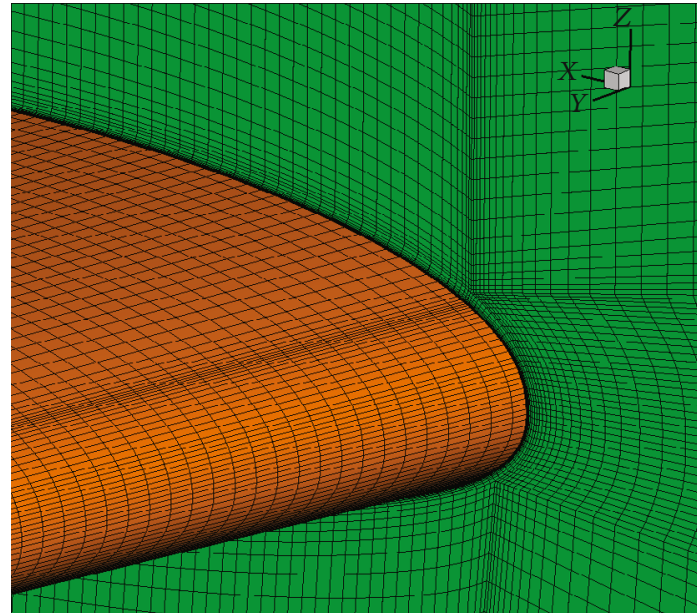

(a) The front of the root

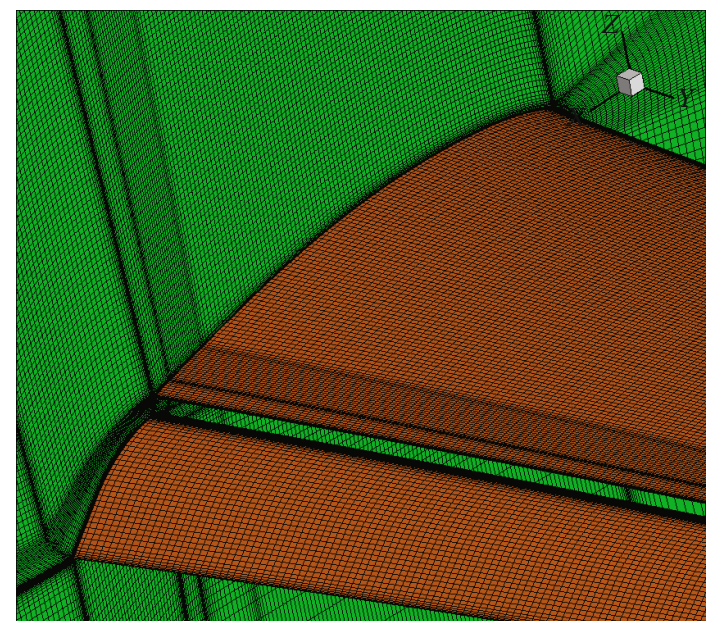

(b) Wing surface and symmetry

FIGURE 7: Illustration of surface grid details.

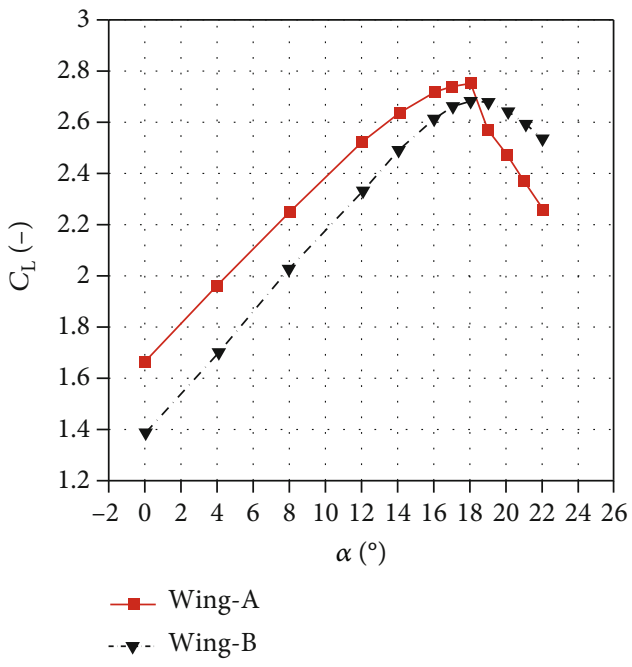

(a) Lift coefficient

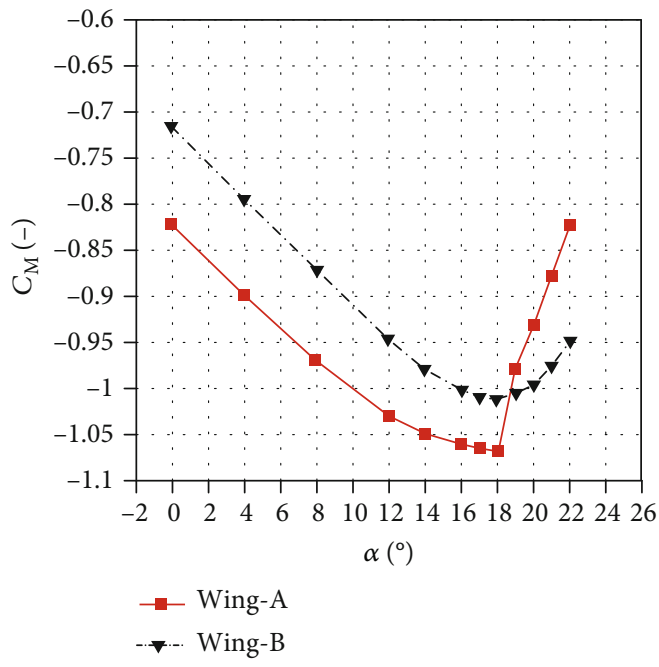

(b) Moment coefficient

FIgURE 8: Comparisons of lift and moment coefficient curves for Wing-A and Wing-B.

that at 18 degrees and gradually decreases to 2.54 at 22 degrees. The absolute value of the pitching moment coefficient depicted in Figure 8(b) has the same changing tendency with the lift coefficient, and the change trend of the moment also reverses at 18 degrees. The rapid reduction of lift and absolute pitching moment of Wing-A after 18 degrees is not conducive to the longitudinal stability and flight safety of the aircraft. In contrast, Wing-B's lift and moment coefficients change gently after an 18-degree angle of attack so that even if the aircraft encounters stall condition, the aerodynamic characteristics will not suddenly deteriorate to provide the pilot enough time to handle this kind of situation and get the plane out of stall.

Figures 9 and 10 show the development of surface streamlines (averaged over 2000 steps) and the distribution of the instantaneous spatial vortex structure of Wing-A and Wing-B at an 18-, 20-, and 22-degree angle of attack, respectively. It can be clearly seen from Figure 9 that the onset and evolution of flow separations over the surface under the stall condition of the two wings are obviously different with each other and this directly results in the discrepancy of stall characteristics between them. For Wing-A, the flow on the suction side separates from the trailing edge in the middle of the main wing when a critical angle of incidence is reached. The separation zone gradually expands as the angle of attack continues to increase to 20 degrees. Then, as it finally reaches 22 degrees, the wing meets a rapid enlargement of the separation zone with a complicated spatial vortex structure and severe flow separation, directly resulting in a sudden decrease in the lift and absolute pitching moment coefficients. As can be seen from the comparison in Figure 9 that the separation process on Wing- $B$ is completely different from that on Wing- $A$, there is no visible flow separation on Wing- $B$ at an 18-degree angle of attack. When it comes to 20 degrees, the separation first starts from the trailing edge near the wing root region and is suppressed during the process of gradually 


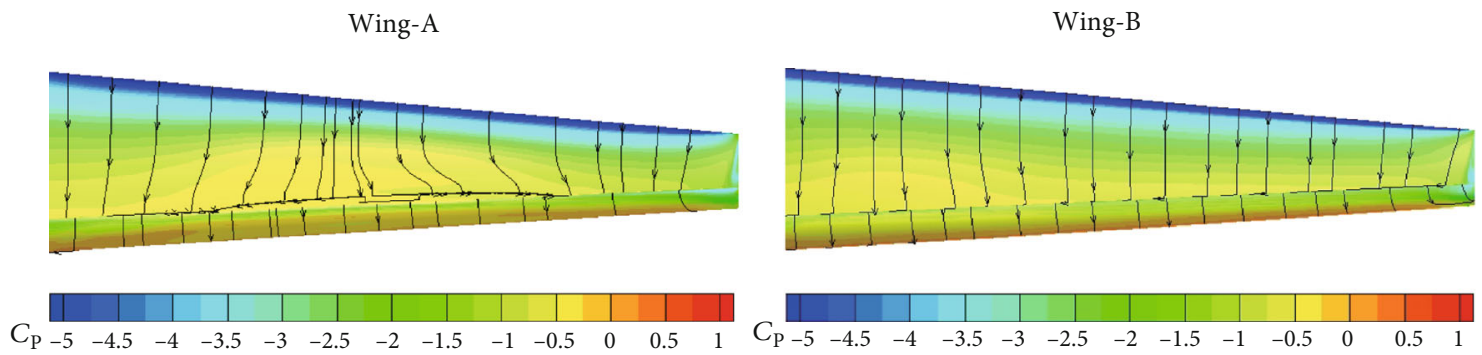

(a) $\alpha=18^{\circ}$

Wing-A

Wing-B

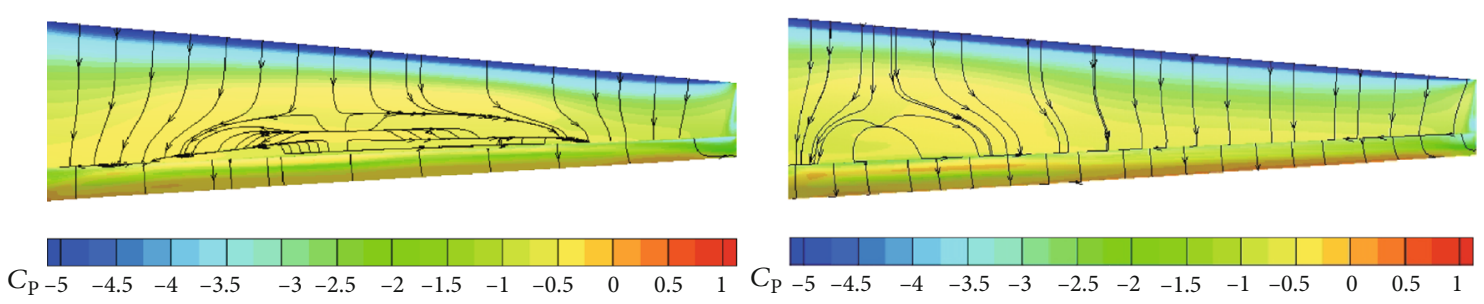

(b) $\alpha=20^{\circ}$

Wing-A

Wing-B
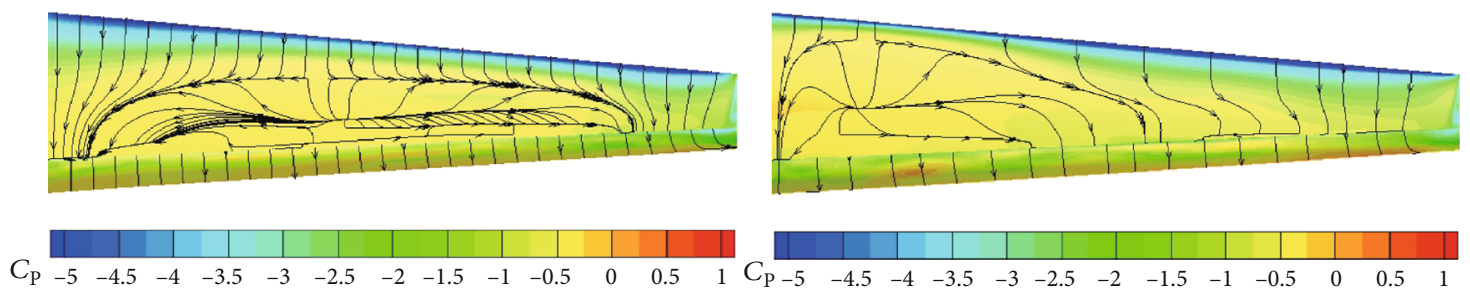

(c) $\alpha=22^{\circ}$

FIGURE 9: Time-averaged pressure contours and surface streamlines at different angles of attack.

expanding towards the wing tip. The outboard wing always maintains attached flow even the angle of attack reaches 22 degrees and the flow separation has occupied the entire inboard wing surface.

An inspection to the instantaneous flow feature contributes to understanding the complexity of unsteady flow around the two-element high-lift configuration. Figure 10 provides a three-dimensional view of the flow field around the high-lift wings. The figure shows turbulent structures defined by an isosurface at a $Q$-criterion which is implemented to identify the spatial structures and the process of vortex generation and development. The isosurface is colored by the streamwise velocity $U$. Comparing Figure 10 (a) with Figures 10(b) and 10(c), there are more turbulent structures present in the flow at a higher angle of attack. This is consistent with the flow separation process of the two wings, and the differences in the separation process between Wing-A and Wing-B are also been clearly reflected. Wing-A starts to separate from the trailing edge in the middle of the main wing at 18 degrees, and the attached flow on the upper surface becomes very unstable, resulting in the existence of many small turbulent structures on the wing surface around the separation zone as well as large-scale vortices in the nearfield wake region. At 20 and 22 degrees, the structures with various scales become highly complex and unsteady and almost occupy the entire wing surface. For Wing-B, there is almost no turbulent structure on the suction side at 18 degrees. As shown in Figure 10(b), the highly unsteady turbulent structures with larger velocity gradients mainly exist at the region near the wing root at 20 degrees. When it comes to 22 degrees, the spatial vortex structures grow in size and further extend in both the chordwise and spanwise directions along the upper surface.

According to the comparison and analysis above, the following basic conclusions can be drawn. Wing-A separates earlier and the separation zone is easier to expand and occupies a larger area of the wing surface. Wing-B is accompanied by flow separation with higher strength above the wing at poststall conditions. The separated flow regions produce a highly unsteady and turbulent flow field mainly consisting of large-scale vortices with larger velocity gradients. However, the separation zone of Wing-B is only limited to the inboard wing area. Therefore, the effect of flow separation on aerodynamic characteristics of Wing- $\mathrm{A}$ after stall is much greater than that of Wing-B in general and this is the reason why the aerodynamic characteristics of Wing-A change dramatically while the changing process is relatively mild for Wing-B. 

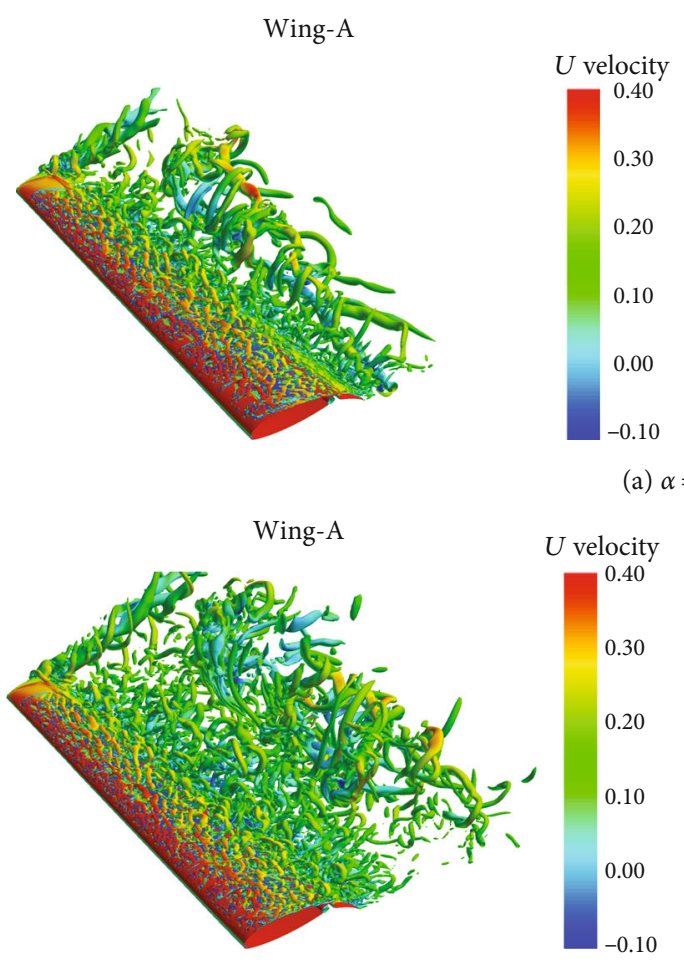

Wing-B
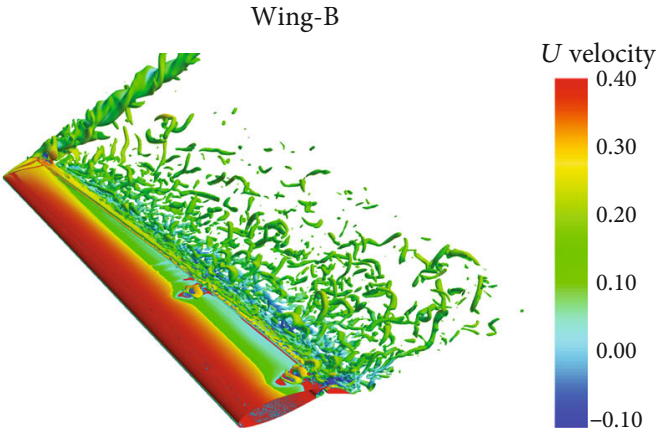

(a) $\alpha=18^{\circ}$

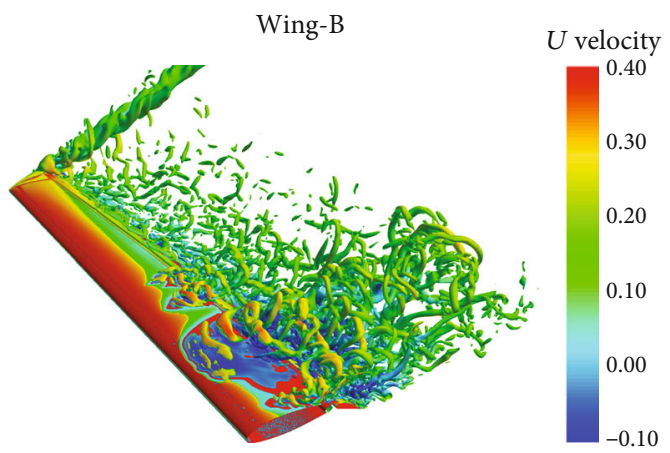

(b) $\alpha=20^{\circ}$
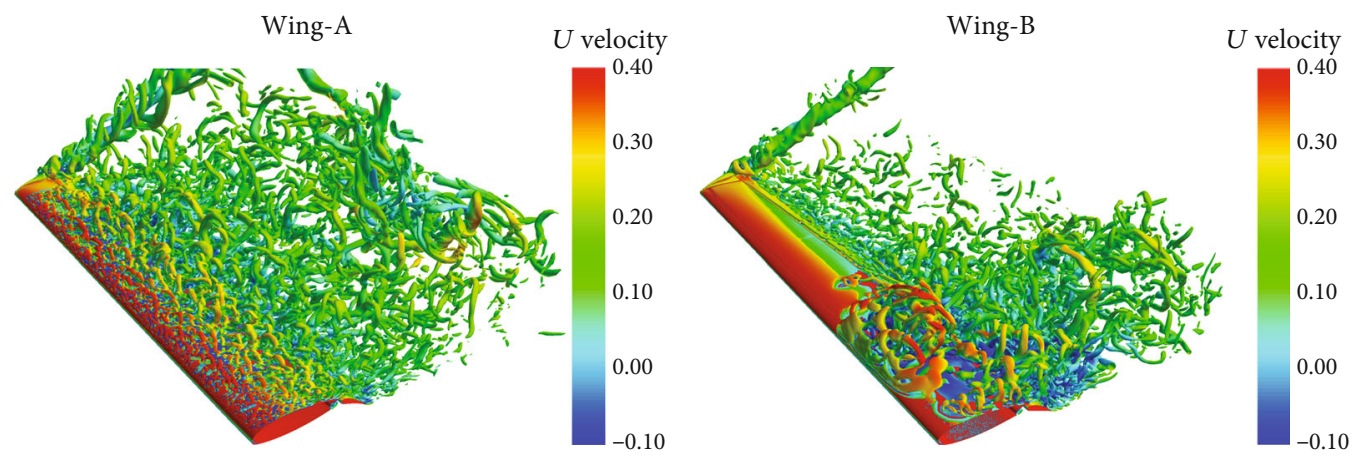

(c) $\alpha=22^{\circ}$

FIGURE 10: Comparison of instantaneous flow topology based on a $Q$-criterion colored by the streamwise velocity $U$ around wings at different angles of attack.

\section{An Efficient Method for Assessment and Improvement of Stall Characteristics}

The IDDES method has been successfully applied for the investigations on stall characteristics of wing and high-lift configurations in previous studies. However, it is still too time-consuming to be used in a design loop where rounds of repetitive computations are required. As an alternative to IDDES, an efficient method which only involves twodimensional RANS calculations is proposed for the assessment and improvement of stall characteristics in this section.

4.1. The Relevance of Stall Characteristics between High-Lift Configuration and Wing Sections. After a detailed investigation on the flow field of high-lift configurations of Wing-A and Wing-B at poststall conditions, conclusion can be made that the stall characteristics of the wings are directly related to the onset and development of separation on the suction side. So, in order to clarify which part of the configuration dominates the separation process on the wing surface, additional simulations of the clean wing of Wing-A and Wing-B are implemented using the same hybrid method. The comparison of the lift coefficient for clean wings and high-lift configurations of Wing-A and Wing-B is described in Figure 11. We can see that the stall characteristics of the two configurations of Wing-A or Wing-B are basically consistent no matter with or without the high-lift device. The high-lift system directly causes a significant increase in lift and may lead to the change of the critical angle of attack within a limited range. As shown in Figure 11, the stall angle of the clean wing of Wing-B decreases from 20 to 18 degrees after opening the high-lift device. Although the stall angle changes, there will be no fundamental change in the stall pattern of the wing due to the high-lift mechanism. 


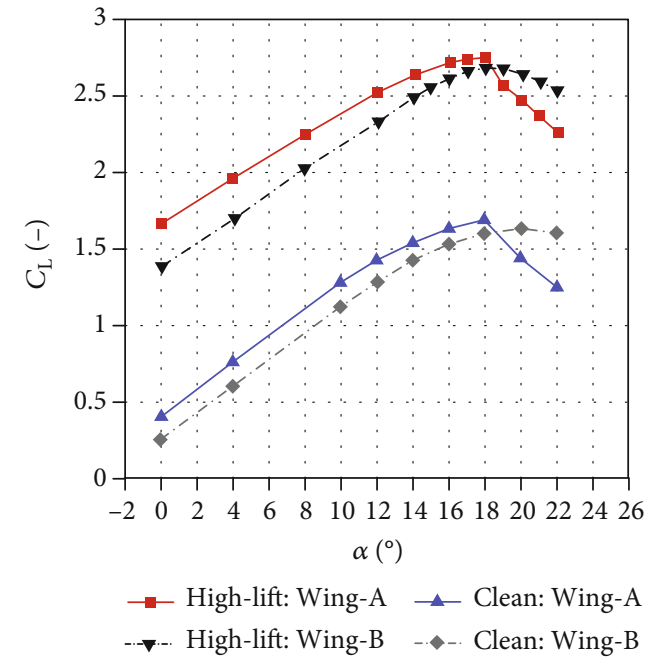

Figure 11: Comparison of the lift coefficient for the clean wing and high-lift configuration of Wing-A and Wing-B.

TABLE 4: Local Reynolds number of each wing section.

\begin{tabular}{lcccc}
\hline Wing section & 1 & 2 & 3 & 4 \\
\hline $\operatorname{Re}_{\mathrm{L}}\left(\times 10^{6}\right)$ & 13.60 & 10.90 & 9.00 & 5.36 \\
\hline
\end{tabular}

For wings, airfoil profiles at different sections become the main factors affecting the aerodynamic characteristics once the sizes and layout of the wings are given. Hence, the relevance of stall characteristic between the three-dimensional configuration and two-dimensional airfoil profile of each section is further discussed. The aerodynamic characteristics of four airfoil profiles are calculated by a RANS solver, and the stall angle of each section is achieved. Although the RANS method is not suitable for predicting the aerodynamic characteristics of airfoils after stall, its prediction of the stall point is credible [25]. Table 4 gives the local Reynolds number based on the chord length of each wing section. The stall angle distribution of the four sections for both the clean wing and high-lift configurations of Wing-A and Wing-B along the wingspan is depicted in Figure 12, revealing the association between the flow separation characteristics and stall angle distribution of sections along the span. The flow separation on the wing surface tends to start at the location near the section with a minimum critical angle of attack, and the evolution of separation is up to the distribution of the stall angle. As shown in Figure 12, the curves of stall angle distribution of Wing-A seem to be the cross section of an asymmetric plate while those of Wing-B look like a "tick." For Wing- $A$, there is a little difference in stall angles of these wing sections. The stall angles of Wing-A's high-lift configuration are between 14 and 15 degrees, and the value at sections 2 and 3 are both 14 degrees, just like the "bottom of the plate." Hence, flow separation is prone to start at the middle of the wing, and the separation zone on the suction side will not meet too much resistance during the process of expansion. For Wing- $\mathrm{B}$, the stall angle of section 2 is a minimum, just like the "kink of the tick." The stall angle of section 2 is

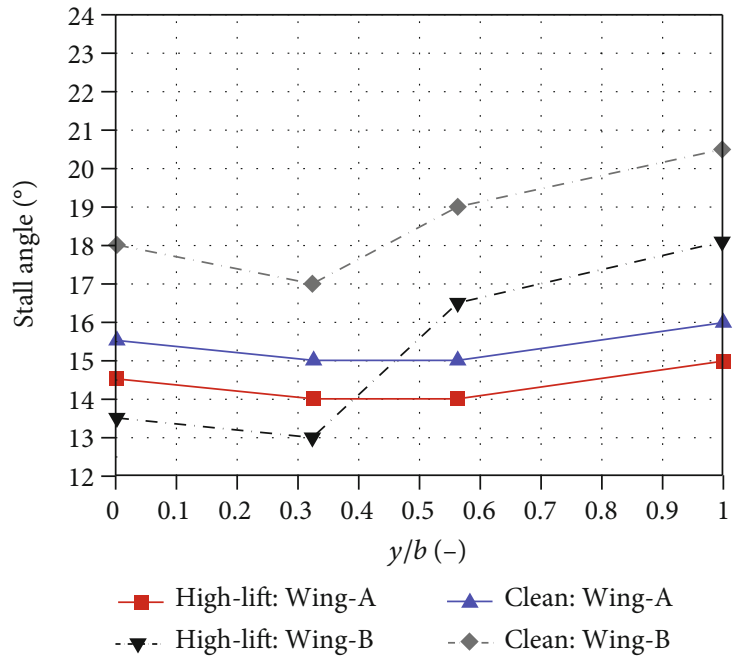

FIGURE 12: Stall angle distribution along the wingspan for the clean wing and high-lift configuration of Wing-A and Wing-B.

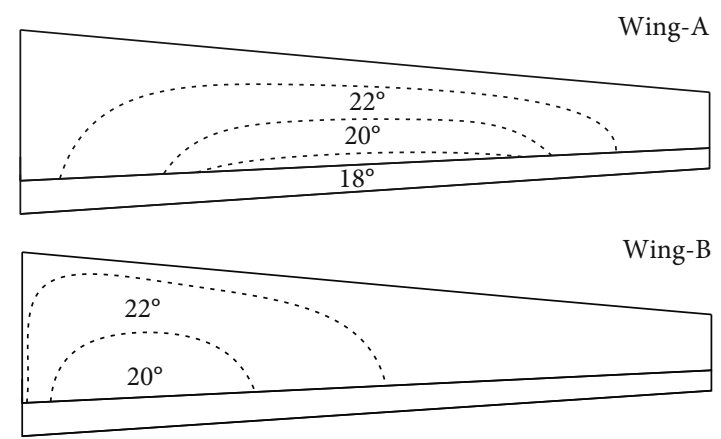

FIGURE 13: Stall pattern: contour lines indicate areas of separated flow at the angle of attack shown.

slightly smaller than that of section 1 , but it changes from section 2 to the wingtip with a considerable increment. Therefore, the flow on the upper surface between sections 1 and 2 prefers to separate first of all, and the separation zone is mainly restricted at the inboard part of the wing as the development of flow separation to the wing tip is strongly suppressed. The development process of flow separation predicted by wing sections' stall angle distribution is consistent with the wing stall pattern depicted in Figure 13 obtained from time-averaged surface streamlines of IDDES results in Figure 9.

Previous numerical studies have demonstrated that there is a significant correlation between the stall angle distribution along the wingspan and flow separation pattern. The flowchart of the present efficient evaluation system is described in Figure 14. First, the stall points of two-dimensional wing sections are achieved by RANS computation. And then, the stall angle distribution along the span will serve as the criteria for qualitative evaluation of the stall pattern. The predicted results which are consistent with those of IDDES can be used as design feedback to engineers for the next round of improvements. 


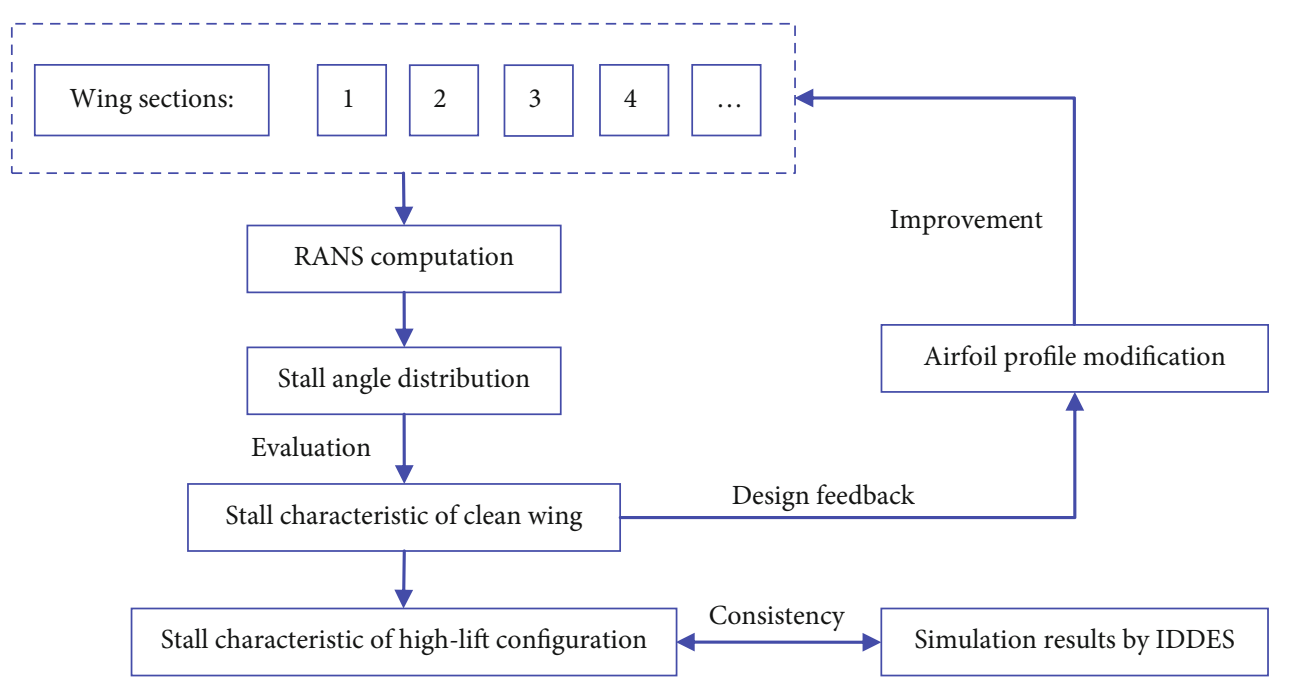

FIGURE 14: Flowchart of the efficient evaluation system.

4.2. Guidelines for the Design of Mild-Stall High-Lift Configuration without Slats. According to the comparison and analysis above, several constructive guidelines which are beneficial to the design of mild-stall high-lift configuration without slats are proposed as follows:

(1) From the perspective of aerodynamic design, the key to improving the stall characteristics of aircraft is to effectively control the onset and development of flow separation. The stall progression on the wing should be controlled to avoid the dramatic change of aerodynamic coefficients and provide natural buffet stall warning. The flow separation should always be kept in the inboard wing because once it occurs outboard on the wing, lateral controllability deteriorates and roll damping is reduced [9]

(2) The good stall pattern depends on judicious selection of airfoil sections, proper combinations of wing thickness, twist, etc. [9]. The results of Wing-A indicate the fact that high-lift airfoils are not suitable for two-element high-lift configuration especially for sections at the middle or outer part of the wing because of its poor control of the leading-edge pressure peak at a high angle of attack. Although a wing with high-lift airfoils can easily provide adequate lift during takeoff and landing, its stall characteristic is unacceptable

(3) The stall pattern of two-element high-lift configuration can be changed by adjusting the stall angle distribution of wing sections. An ideal distribution of a local stall angle from the wing root to tip should look like a "tick" because the great increment in the stall angle along the span is able to control the evaluation of flow separation and prevent complete stalling of the wing

(4) The local critical angle of attack of different control sections and their distribution can be adjusted through the modification of the geometrical features of airfoil profiles. The leading-edge bluntness, cam- ber, and upper-surface geometry can all be used to control the pressure peak on the suction side and thereby adjust the initial angle of attack where airfoil separation appears. NASA has conducted a large number of experiments to investigate the influence of airfoil geometric parameters on its aerodynamic and stall characteristics before [26, 27], and lots of valuable conclusions have been obtained

\section{Conclusion}

In this study, numerical simulations of CRM under poststall conditions have been conducted using both the URANS and IDDES models. As a newly developed CFD method, IDDES is able to provide rich flow field details and complete force results which cannot be easily obtained through a wind tunnel experiment or flight test. Hence, IDDES can be used as a state-of-the-art CFD tool for the investigation on stall characteristics of complex high-lift configurations with different geometric characteristics to better understand the physics of onset, evolution, and effect of flow separation under poststall conditions.

In order to meet the flight safety requirements of aircraft during the process of takeoff and landing, the stall characteristics of the aircraft should be relatively gentle. That is to say, when the angle of attack of the aircraft is greater than the critical angle of attack, flow separation on the wing surface should be a controllable process and large areas of sudden separation should be avoided. From the perspective of aircraft's controllability and stability, flow separation should start from the inboard region of the wing and always has less impact on the outboard wing to avoid the loss of control surface effectiveness.

From the research in Section 4, it can be concluded that the onset and development of flow separation on the upper surface are mainly dominated by the profiles and the stall angle distribution of two-dimensional airfoils at different sections along the span. Hence, the qualitative analysis and evaluation of stall characteristic of three-dimensional 
configuration can be simply carried out by getting the stall angle distribution of those sections without excessive time and computational resource consumption. Based on this, an efficient evaluation method that is capable of predicting and improving the stall performance of wings in the preliminary stage of design is formed and several design rules for mild-stall two-element high-lift configurations are proposed as follows:

(1) The stall performance of two-element high-lift configuration mainly depends on that of the clean wing as the high-lift mechanism of the flap can hardly bring any fundamental change to the clean wing's stall characteristics. Therefore, great importance should be attached to the design of basic clean wing configuration at the first stage

(2) High-lift airfoils are not suitable for sections at the middle or outboard part of configuration without slats as they may cause premature flow separation on the outboard wing and deterioration of stall characteristics due to their poor control of the leadingedge pressure peak at a high angle of attack

(3) In order to avoid the effectiveness reduction of control surfaces, the flow separation should start from the inboard region and will not expand to the outboard wing in a short time after stall

(4) The development of flow separation on the wing surface should be a controllable process, and large areas of sudden separation should be avoided to prevent the dramatic change of aerodynamic characteristics

(5) The stall pattern of two-element high-lift configuration can be changed by adjusting the stall angle distribution of wing sections. An ideal distribution of a local stall angle from the wing root to tip should look like a "tick"

\section{Abbreviations}

CFD: $\quad$ Computational fluid dynamics

CRM: Common Research Model

ETW: $\quad$ European Transonic Wind Tunnel

IDDES: Improved delayed detached eddy simulation

LES: $\quad$ Large eddy simulation

RANS: Reynolds-averaged Navier-Stokes

URANS: Unsteady Reynolds-averaged Navier-Stokes

WMLES: Wall-modeled large eddy simulation

$C_{\mathrm{D}}: \quad$ Drag coefficient

$C_{\mathrm{L}}: \quad$ Lift coefficient

$C_{\mathrm{M}}: \quad$ Pitching moment coefficient

$C_{\mathrm{P}}$ : $\quad$ Pressure coefficient

Ma: $\quad$ Mach number

Re: $\quad$ Reynolds number

$x / b$ : $\quad$ Distance from the wing root in the $X$ direction nondimensionalized by the half wing span

$x / c: \quad$ Distance from the wing leading edge in the $X$ direction nondimensionalized by the local wing chord

$\begin{array}{ll}y / b: & \text { Distance from the wing root in the } Y \text { direction } \\ & \text { nondimensionalized by the half wing span } \\ z / c: & \text { Coordinate value in the } Z \text { direction nondimen- } \\ & \text { sionalized by the local wing chord } \\ \alpha: & \text { Angle of attack (degree) } \\ \eta: & \text { Fraction of the wing semispan. }\end{array}$

\section{Data Availability}

The data used to support the findings of this study are included within the article.

\section{Conflicts of Interest}

The authors declare that they have no conflicts of interest.

\section{References}

[1] M. P. Patel, Z. H. Sowle, T. C. Corke, and C. He, "Autonomous sensing and control of wing stall using a smart plasma slat," Journal of Aircraft, vol. 44, no. 2, pp. 516-527, 2007.

[2] C. P. van Dam, "The aerodynamic design of multi-element high-lift systems for transport airplanes," Progress in Aerospace Sciences, vol. 38, no. 2, pp. 101-144, 2002.

[3] D. Sasaki, A. Ito, T. Ishida, and K. Nakahashi, "Design optimization of a mild-stall airfoil/wing for UAV and PAV applications," in 27th AIAA Applied Aerodynamics Conference, San Antonio, TX, USA, 2009.

[4] A. Moitra, P. Gujar, P. Gupta, and D. Rajvanshi, "CFD evaluation of the effects of stall control devices and transition tapes on stall behavior of airplanes," in 32nd AIAA Applied Aerodynamics Conference, Atlanta, GA, USA, 2014.

[5] P. Reynolds, "Ten years of stall testing," in Orbital Debris Conference: Technical Issues and Future Directions, Baltimore, MD, USA, 1990.

[6] J. L. Johnson Jr., W. A. Newsom, and D. R. Satran, "Full-scale wind-tunnel investigation of the effects of wing leading-edge modifications on the high angle-of-attack aerodynamic characteristics of a low-wing general aviation airplane," in AIAA Aircraft System Meeting, Anaheim, CA, USA, 1980.

[7] H. F. Meyer, L. P. Yip, J. N. Perkins, and R. J. Vess, "Experimental investigation of the high angle of attack characteristics of a high performance general aviation aircraft," in 7th Applied Aerodynamics Conference, Seattle, WA, USA, 1989.

[8] A. Winkelmann, J. Barlow, J. Saini, J. Anderson Jr., and E. Jones, "The effects of leading edge modifications on the post-stall characteristics of wings," in 18th AIAA Aerospace Sciences Meeting, Pasadena, CA, USA, 1980.

[9] S. B. Anderson, "Historical overview of stall/spin characteristics of general aviation aircraft," Journal of Aircraft, vol. 16, no. 7, pp. 455-461, 1979.

[10] J. R. Forsythe, K. D. Squires, K. E. Wurtzler, and P. R. Spalart, "Detached-eddy simulation of the F-15E at high alpha," Journal of Aircraft, vol. 41, no. 2, pp. 193-200, 2004.

[11] S. A. Morton, M. B. Steenman, and R. M. Cummings, "DES grid resolution issues for vortical flows on a delta wing and an F-18C," in 41st Aerospace Sciences Meeting and Exhibit, Reno, NV, USA, 2003.

[12] J. R. Forsythe and S. H. Woodson, "Unsteady computations of abrupt wing stall using detached-eddy simulation," Journal of Aircraft, vol. 42, no. 3, pp. 606-616, 2005. 
[13] M. Tomac, A. Jirasek, and A. Rizzi, "Hybrid Reynoldsaveraged Navier-Stokes/large-eddy simulations of F-16XL in low-speed high-alpha flight," Journal of Aircraft, vol. 54, pp. 2070-2076, 2017.

[14] S. Fu, Z. Xiao, H. Chen, Y. Zhang, and J. Huang, "Simulation of wing-body junction flows with hybrid RANS/LES methods," International Journal of Heat and Fluid Flow, vol. 28, no. 6, pp. 1379-1390, 2007.

[15] Z. Xiao, H. Chen, Y. Zhang, J. Huang, and S. Fu, "Study of delayed-detached eddy simulation with weakly nonlinear turbulence model," Journal of Aircraft, vol. 43, no. 5, pp. 13771385, 2006.

[16] A. Waldmann, P. P. Gansel, T. Lutz, and E. Kramer, "Unsteady wake of the NASA Common Research Model in low-speed stall," Journal of Aircraft, vol. 53, no. 4, pp. 1073-1086, 2016.

[17] T. Lutz, P. P. Gansel, A. Waldmann, D.-M. Zimmermann, and S. S. a. Hülse, "Prediction and measurement of the common research model wake at stall conditions," Journal of Aircraft, vol. 53, no. 2, pp. 501-514, 2016.

[18] D. M. Zimmermann, A. Waldmann, T. Lutz, and E. Kramer, "Development of flow structures in the near-field wake region of the common research model," CEAS Aeronautical Journal, vol. 9, no. 2, pp. 347-359, 2018.

[19] J. A. Escobar, C. A. Suarez, C. Silva, O. D. López, J. S. Velandia, and C. A. Lara, "Detached-eddy simulation of a wide-body commercial aircraft in high-lift configuration," Journal of Aircraft, vol. 52, no. 4, pp. 1112-1121, 2015.

[20] M. L. Shur, P. R. Spalart, M. K. Strelets, and A. K. Travin, “A hybrid RANS-LES approach with delayed-DES and wallmodelled LES capabilities," International Journal of Heat and Fluid Flow, vol. 29, no. 6, article S0142727X08001203, pp. 1638-1649, 2008.

[21] M. Rivers, J. Quest, and R. Rudnik, "Comparison of the NASA Common Research Model European transonic wind tunnel test data to NASA national transonic facility test data," CEAS Aeronautical Journal, vol. 9, pp. 307-317, 2018.

[22] T. Lutz, Going for Experimental and Numerical Unsteady Wake Analyses Combined with Wall Interference Assessment by Using the NASA CRM-Model in ETW, American Institute of Aeronautics and Astronautics, 2013.

[23] M. Rivers, C. Hunter, and R. Campbell, "Further investigation of the support system effects and wing twist on the NASA Common Research Model," in 30th AIAA Applied Aerodynamics Conference, New Orleans, Louisiana, 2012.

[24] L. Zhang, J. Li, Y. F. Mou, H. Zhang, W. B. Shi, and J. Jin, "Numerical investigation of flow around a multi-element airfoil with hybrid RANS-LES approaches based on SST model," Journal of Mechanics, vol. 34, pp. 123-134, 2018.

[25] D. Li, I. Men'shov, and Y. Nakamura, "Detached-eddy simulation of three airfoils with different stall onset mechanisms," Journal of Aircraft, vol. 43, no. 4, pp. 1014-1021, 2006.

[26] C. A. Szelazek and R. M. Hicks, "Upper-Surface Modifications for $\mathrm{c}(\mathrm{l}(\max ))$ Improvement of Selected NACA 6-Series Airfoils," 1979, NASA TM-78603.

[27] R. M. Hicks and E. T. Schairer, "Effects of upper surface modification on the aerodynamic characteristics of the NACA 63(2)-215 airfoil section," 1979, NASA-TM-78503. 


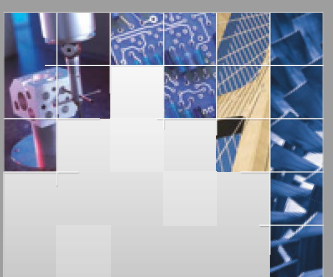

\section{Enfincering}
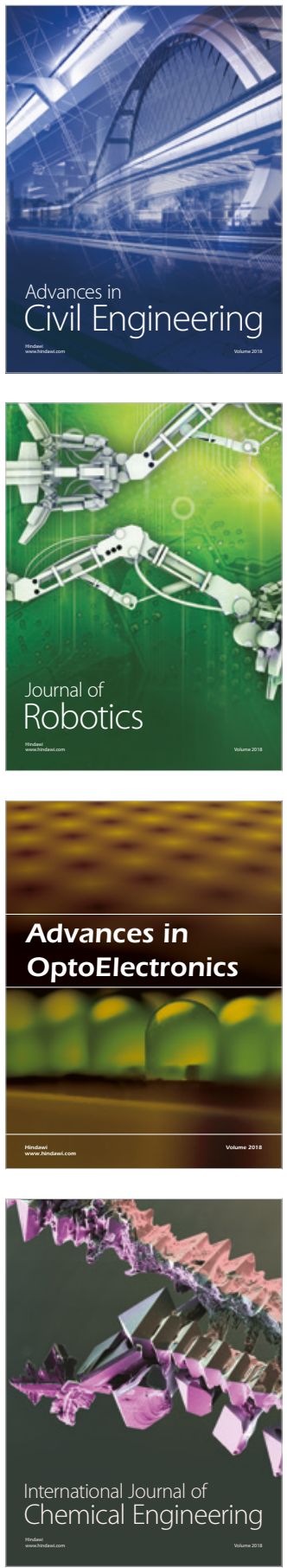

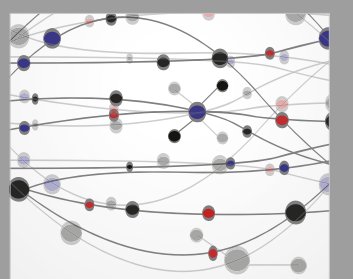

\section{Rotating \\ Machinery}

The Scientific World Journal

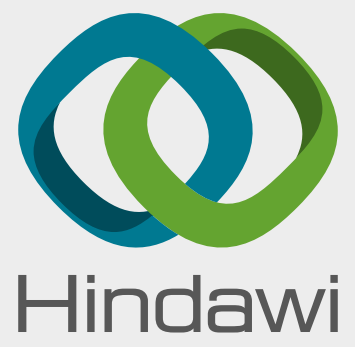

Submit your manuscripts at

www.hindawi.com
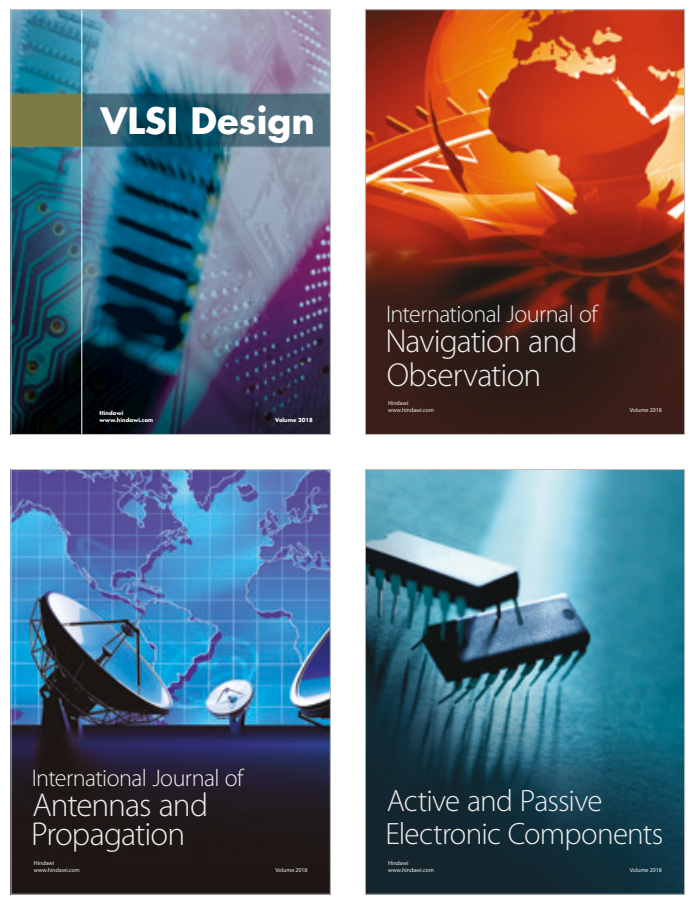
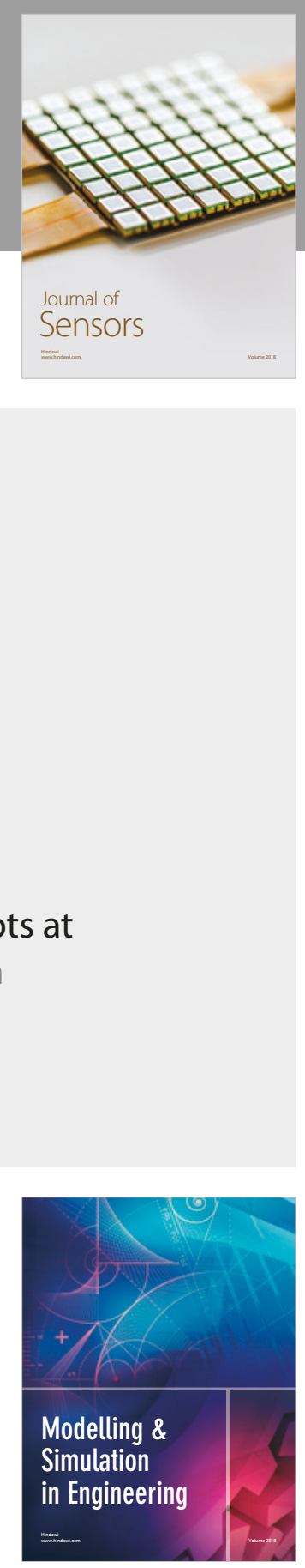

\section{Advances \\ Multimedia}
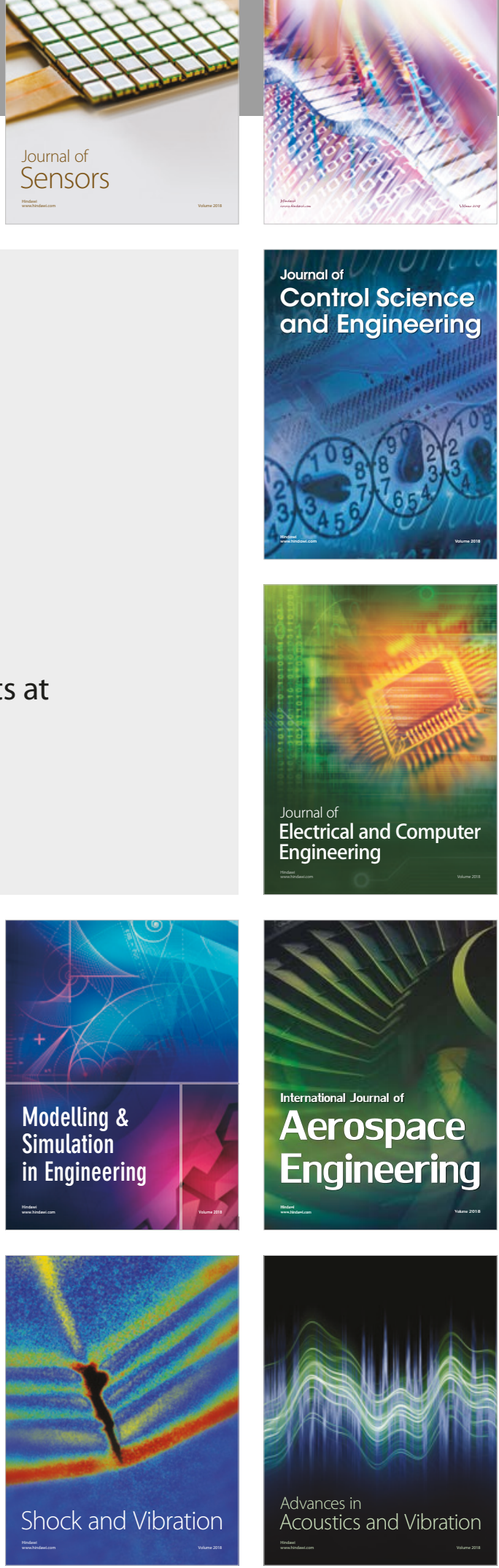